\title{
Venlafaxina retard y trastorno por dependencia de alcohol
}

\author{
David Cáceres Anillo*; Ana Rodriguez Yuste**; Carlos Morillo Velarde***; Ma Victoria Cabrera \\ GISBERT****
}

Unidad de desintoxicación Hogar Renacer de Córdoba,

*Director Médico, **Psicóloga, ***Psiquiatra, *** Médico

Enviar correspondencia a:

David Cáceres Anillo. Unidad de desintoxicación Hogar Renacer de Córdoba, C/ Juan Felipe de Vilela n¹8. Córdoba.

david.caceres@hogar-renacer-cordoba.com

\section{RESUMEN}

Objetivos: Determinar el efecto del tratamiento con venlafaxina retard en pacientes con trastorno por dependencia de alcohol o de alcohol y cocaína que inician un tratamiento de desintoxicación.

Metodología: Estudio observacional, abierto y prospectivo, realizado en España en 2005. Se incluyen 55 pacientes mayores de 18 años con diagnóstico de trastorno por dependencia de alcohol o alcohol y cocaína, ingresados en un centro de Atención Especializada para iniciar tratamiento de desintoxicación. Se administró durante 6 meses venlafaxina retard, a dosis entre 75 y 225 mg/día.

Resultados: El tratamiento se asoció a reducciones significativas en las puntuaciones del EuropASI en las siguientes áreas: 3, uso de alcohol, con una puntuación basal y final de $8,2 \pm 0,2$ y $6,4 \pm 0,4$, respectivamente $(P<0,01)$; 5 , relaciones familiares/sociales, puntuación inicial de 6,9 $\pm 0,2$ y final de 5,2 $\pm 0,5(P<0,001) ; 1$, situación médica, con puntuaciones de 3,7 $\pm 0,4$ y $0,9 \pm 0,3$ (visitas basal y final, respectivamente) $(P<$ $0,001)$; y 6 , situación psiquiátrica, con puntuación basal de 7,8 $\pm 0,1$ y final de 5,4 $\pm 0,4(P<0,001)$. La puntuación basal en la Escala Visual Analógica de craving de alcohol fue de 26,7 $\pm 4,6$, descendiendo a $4,1 \pm 1,5$ en la visita final $(P<0,001)$.

Conclusiones: Los resultados de este estudio observacional sugieren que venlafaxina retard podría ser efectiva en el tratamiento coadyuvante de pacientes dependientes de alcohol, que están siguiendo terapia de deshabituación. No obstante, esto debe ser replicado con series más amplias y controladas con placebo.

Palabras clave: dependencia de alcohol, venlafaxina retard, observacional, desintoxicación.

\section{ABSTRACT}

Objectives: The aim is to determine the effect of the treatment with venlafaxine extended release in patients with alcohol or cocaine dependence disorder that initiate detoxification treatment.

Methods: Observational, open, prospective study carried out in Spain in 2005. 55 patients older than 18 years of age with diagnosis of alcohol and/or cocaine dependence disorder, hospitalized in Specialty Care Center to initiate detoxification treatment, were included. Daily doses of 75 to $225 \mathrm{mg}$ of venlafaxine extended release were administered for 6 months.

Results: Treatment was associated with significant reductions in EuropASI scores in the following areas: 3 , alcohol use, baseline and final score of $8.2 \pm 0.2$ and $6.4 \pm 0.4$, respectively $(P<0.01) ; 5$, family/social relations, initial score of $6.9 \pm 0.2$ and of $5.2 \pm 0.5$ at endpoint $(P<0.001)$; 1 , medical status, scores of $3.7 \pm 0.4$ and $0.9 \pm 0.3$ (baseline and final visits, respectively) $(P<0.001)$; and 6 , psychiatric status, with a baseline score of $7.8 \pm 0.1$ and final score of $5.4 \pm 0.4(P<0.001)$. The VAS alcohol craving scores at baseline were $26.7 \pm 4.6$, decreasing to $4.1 \pm 1.5$ at endpoint $(P<$ 0.001)

Conclusions: The results of this observational study suggest that venlafaxine extended release could be effective as a coadyuvant in the treatment of alcohol dependent patients in alcohol detoxification therapy. Nevertheless, this should be confirmed with bigger placebo-controlled samples.

Key words: Alcohol dependence, venlafaxine extended release, observational desintoxication.

\section{INTRODUCIÓN}

$\mathbf{L}$ os problemas relacionados con el alcohol, incluyendo consumo de riesgo, abuso de alcohol y dependencia de alcohol son altamente prevalentes. La dependencia fisiológica de alcohol se demuestra por la aparición de tolerancia o de síntomas de abstinencia. Los problemas relacionados con él, tienen un notable impacto sobre la mortalidad y morbilidad de los pacientes'. Estudios realizados refieren una mortalidad anual atribuida al alcohol en España del $4,6 \%$ con respecto al total de causas de muerte, con distribución dispar según el sexo. A esto hay que añadir los recursos y el coste que provoca el tratamiento 
de los problemas somáticos y psíquicos generados por su consumo. No se debe olvidar que el paciente alcohólico precisa un número elevado de cuidados médico-quirúrgicos ${ }^{2,3}$. La cocaína puede dar lugar, al igual que el alcohol, a dependencia tras su consumo durante cortos periodos de tiempo'.

El uso de antidepresivos en el tratamiento de la dependencia de alcohol, incluso en aquellos pacientes con síntomas depresivos manifiestos, es un tema de debate que permanece aún sin resolver. La venlafaxina es un inhibidor de la recaptación de serotonina y noradrenalina que ha demostrado su eficacia en el trastorno depresivo ${ }^{4,5}$, en trastorno de ansiedad generalizada (TAG)6, 7, en depresión con $\mathrm{TAG}^{8}$ y en depresión con síntomas asociados de ansiedad ${ }^{9-11}$. Existen tres estudios abiertos con un número reducido de pacientes en los que se evaluó el uso de venlafaxina en el manejo de individuos alcohólicos o con trastornos por uso de sustancias. En el primero de los estudios se observaron importantes reducciones en el uso comunicado de cocaína y se objetivó una disminución en la tasa de positivos en las analíticas realizadas a los pacientes cocainómanos deprimidos ${ }^{12}$. En el segundo estudio analizado se obtuvieron efectos mixtos en el uso de cocaína, así como mejoría en los síntomas del trastorno por déficit de atención e hiperactividad que presentaban los pacientes de forma concomitante ${ }^{13}$. En el tercer estudio revisado en pacientes alcohólicos deprimidos, se observó también una mejoría tanto en la sintomatología anímica como en el consumo de alcohol $^{14,15}$. Destacar que en estos tres estudios abiertos la venlafaxina se toleró bien. Un cuarto estudio en pacientes cocainómanos no deprimidos muestra como la venlafaxina disminuye los efectos subjetivos de la cocaína en un 10 - 20\% de los individuos ${ }^{16}$.

Con este estudio se pretende determinar el efecto coadyuvante del tratamiento con venlafaxina retard en el manejo de pacientes diagnosticados de trastorno por dependencia de alcohol (o de alcohol y cocaína) que inician un tratamiento de desintoxicación. Como objetivos del mismo se pretendió determinar la efectividad del fármaco para reducir la severidad de la dependencia de alcohol (o de alcohol y cocaína), así como el deseo o necesidad de consumo. También se analiza la seguridad y tolerancia del fármaco.

\section{MÉTODOS}

Estudio de carácter observacional, abierto y prospectivo, realizado en España en 2005. Se incluyen en el mismo a pacientes adultos con edad superior a 18 años con diagnóstico de trastorno por dependencia de alcohol o de alcohol y cocaína (criterios DSM-IV) ${ }^{17}$, ingresados en un centro de Atención Especializada para iniciar un tratamiento de desintoxicación. Este centro dispone de una Unidad de Desintoxicación para realización de tratamientos en régimen de ingreso y es atendido por un equipo técnico multidisciplinar. Así mismo, está dotado de consultas externas para el posterior seguimiento de los enfermos en régimen ambulatorio, consultas de enfermería para realización de controles toxicológicos y salas de terapia para el desarrollo de las actividades grupales contempladas en el programa de deshabituación.

Dado el carácter no intervencionista del estudio, se consideran criterios de exclusión los que vienen reflejados en el apartado de contraindicaciones de la ficha técnica de la especialidad farmacéutica: hipersensibilidad conocida a la venlafaxina o uso de inhibidores de la monoamino-oxidasa en los 14 días previos al reclutamiento de los pacientes. Se tuvieron en cuenta las indicaciones reflejadas en los apartados de advertencias, consideraciones especiales e interacciones.

Todos los pacientes recibieron durante 6 meses venlafaxina retard, a dosis entre 75 y 225 mg/día, conforme con las especificaciones indicadas en la ficha técnica de la especialidad farmacéutica y con el criterio clínico del investigador, teniendo en cuenta la severidad del cuadro clínico. Se realizó una visita basal y 5 visitas de seguimiento a los días 15 y mes $1^{\circ}, 2^{\circ}, 3^{\circ}$ y $6^{\circ}$ del estudio. Dado que el tiempo medio de ingreso hospitalario para el inicio y consolidación del tratamiento de desintoxicación fue de 1 mes, la mayoría de las visitas programadas para los meses $2^{\circ}$, $3^{\circ}$ y $6^{\circ}$ se realizaron de manera ambulatoria. El estudio se realizó conforme a los principios éticos contenidos en la Declaración de Helsinki y enmiendas posteriores, normas de Buena Práctica Clínica (BPC), y otras normas internacionales aplicables para la realización de ensayos clínicos en humanos. Los pacientes otorgaron su consentimiento para participar en el estudio, garantizándose en todo momento la confidencialidad de los datos.

\section{Parámetros de evaluación}

Para evaluar el efecto ejercido por venlafaxina retard sobre la severidad de la adicción a alcohol (o a alcohol y cocaína) se determinaron y compararon en el momento basal y el mes $6^{\circ}$ del estudio las puntuaciones medias obtenidas mediante el EuropASI. El EuropASI (versión europea del Índice de la Severidad de la Adicción) es una entrevista semiestructurada y estandarizada diseñada para evaluar la gravedad de la adicción, definida ésta como la necesidad de tratamiento ${ }^{18,19}$. Evalúa, además del consumo de alcohol y otras drogas, otras áreas de la vida del paciente que pueden estar relacionadas con su adicción. Estas áreas son: situación médica, situación laboral y soportes, relaciones familiares y sociales, problemas lega- 
les y estado mental. Desde el punto de vista clínico proporciona información de utilidad para describir las necesidades de los pacientes al inicio de tratamiento, asignar a los pacientes las estrategias terapéuticas adecuadas y evaluar los resultados de las intervenciones terapéuticas.

Para determinar el deseo o necesidad de consumo de alcohol o cocaína, también denominado fenómeno "craving", se evaluaron y compararon las puntuaciones medias obtenidas en la escala Visual Analógica $(E V A)^{20,21}$. Esta escala se utilizó en el estudio, al considerarse un método simple y sensible de medir efectos subjetivos del alcohol y de la cocaína (visita basal, día 15 y mes $1^{\circ}, 2^{\circ}, 3^{\circ}$ y $6^{\circ}$ ). En todas las visitas del estudio se realizó una evaluación subjetiva (preguntas dirigidas) y objetiva (test de detección de drogas en orina) del consumo de alcohol y cocaína.

Adicionalmente se evaluó el efecto ejercido por venlafaxina retard sobre el estado general del paciente ${ }^{22}$ y sobre los posibles síntomas depresivos asociados. Para ello se utilizaron las escalas de Impresión Clínica Global de Severidad (ICG-S) y de Mejoría (ICG$\mathrm{M})$, conforme a la opinión del médico y del paciente (visita basal, día 15 y mes $1^{\circ}, 2^{\circ}, 3^{\circ}$ y $6^{\circ}$ ) y la escala de Hamilton para la depresión de 17 ítems (HAM-D ${ }_{17}$ ) (visita basal y mes $\left.6^{\circ}\right)^{23-25}$. Los parámetros de seguridad y tolerabilidad del tratamiento con venlafaxina retard incluidos fueron: la determinación del grado de cumplimentación terapéutica, el registro de todas las reacciones adversas acaecidas durante el estudio, valorándose su gravedad y su posible relación con el tratamiento del estudio. Así mismo se monitoriza la frecuencia cardiaca y la tensión arterial.

\section{Análisis estadístico}

Las variables cualitativas se analizaron mediante frecuencias absolutas y relativas. Se ha utilizado el test $\mathrm{Q}$ de Cochran o test de Friedman, para la comparación entre visitas de variables dicotómicas o no dicotómicas discretas, con un nivel de significación $(\mathrm{p}<0.05)$.

Las variables cuantitativas se analizaron mediante las medidas de centralización y dispersión. Se ha utilizado el test de Wilcoxon (Signed Rank Test), con el fin de determinar si existen diferencias estadísticamente significativas entre visitas de dichas variables, con un nivel de significación $(p<0.05)$. Como paquete estadístico se utilizó SAS 9.1.

\section{RESULTADOS}

\section{Características demográficas y basales}

Se analiza una muestra de 55 pacientes adultos con diagnóstico de trastorno por dependencia de alco- hol (o de alcohol y cocaína) (criterios DSM-IV), que fueron ingresados en un centro de atención especializada para iniciar un tratamiento de desintoxicación. La edad media de los pacientes fue de 40,6 1,3 años. Del total de pacientes incluidos, un 69,1\% eran hombres. La totalidad de los pacientes consumían alcohol en la visita basal, con una media de consumo de 23,1 $\pm 1,2$ años. La cantidad de alcohol consumido en el mes previo a su inclusión en el estudio fue de 188,6 \pm $14,5 \mathrm{gr} /$ día y el consumo era diario en el $78,2 \%$ de los casos. En el caso del consumo de cocaína, el 20\% de los pacientes consumían basalmente cocaína, con una edad media de consumo de 25,5 $\pm 2,7$ años. La cantidad de cocaína consumida en el mes previo a su inclusión en el estudio fue de 1,3 \pm 0,3 gr/día, el consumo 1 vez a la semana en el $45,5 \%$ de los casos y la vía de administración intranasal en el 81,8\% de los casos.

El estado general de los pacientes, conforme a la puntuación en la escala ICG-S, fue considerado como moderadamente enfermo en el $12,7 \%$ de los casos, marcadamente enfermo en el $61,8 \%$ y gravemente enfermo en el $25,5 \%$. El 21,8\% de los pacientes presentaban enfermedades concomitantes, siendo las más frecuentes las que afectaban a los sistemas músculo-esquelético, cardiovascular y metabólico (5,4\% sobre el total de pacientes, todos los casos). Los pacientes recibieron en la unidad de desintoxicación la medicación habitual en estos casos, iniciando simultáneamente el tratamiento con venlafaxina retard. Así, un 92,7\% de los pacientes recibieron medicación psicotropa en el momento de la inclusión en el estudio, siendo los ansiolíticos/hipnóticos más frecuentemente administrados clormetiazol, clorazepato y lorazepam (80\%, 49,1\% y $16,4 \%$ sobre el total de pacientes, respectivamente). Entre otros fármacos psicotrópos administrados con menor frecuencia destacan olanzapina $(14,6 \%)$, trazodona $(12,7 \%)$, naltrexona y carbamazepina $(5,4 \%$, en ambos casos) (porcentajes sobre el total de la muestra). Se usó medicación disuasiva para consumo de alcohol: cianamida cálcica $(78,2 \%)$ y disulfiram (7,3\%). Un 89,1\% de los pacientes recibieron concomitantemente otra medicación no psicotropa, siendo los fármacos más frecuentemente administrados tiamina, piracetam y citicolina $(61,8 \%$, $34,6 \%$ y $9,1 \%$, respectivamente). La dosis media diaria de venlafaxina retard administrada en la visita basal fue de 90 mg (rango: 75-225 mg/día; IC 95\%: 80,1 -99,9). En la tabla 1 se resumen las principales características basales de la muestra.

\section{Evaluación de efectividad}

El tratamiento con venlafaxina retard, desde el inicio de la desintoxicación hasta la evaluación de resultados a los 6 meses, se asoció a reducciones significativas en las puntuaciones del EuropASI en las siguientes áreas: área 3, uso de alcohol, con una 
Tabla 1: Características demográficas y basales de la muestra

\begin{tabular}{|c|c|c|}
\hline \multicolumn{2}{|c|}{ VARIABLES EVALUADAS } & \multirow{2}{*}{$\frac{\text { POBLACIÓN DEL ESTUDIO N = } 55}{38(69,1)}$} \\
\hline Género & Hombre, $N^{\circ}(\%)$ & \\
\hline & Mujer, $\mathbf{N}^{\circ}(\%)$ & $17(30.9)$ \\
\hline \multicolumn{2}{|c|}{ Edad media; años (rango) ${ }^{a}$} & $40,6 \pm 1,3(20-58)$ \\
\hline \multicolumn{2}{|c|}{ Dependencia de alcohol, Nº $(\%)$} & $55(100)$ \\
\hline \multicolumn{2}{|c|}{ Dependencia de cocaína, Nº (\%) } & $11(20)$ \\
\hline \multicolumn{2}{|c|}{ Enfermedades concomitantes, $\mathrm{N}^{\circ}(\%)$} & $12(21,8)$ \\
\hline \multirow{4}{*}{$\begin{array}{l}\text { Medicación } \\
\text { concomitante }\end{array}$} & Psicotropa, $\mathrm{N}^{\circ}(\%)$ & $51(93,7)$ \\
\hline & - Ansiolíticos/hipnóticos & $49(89,1)$ \\
\hline & - Otros & $28(50,9)$ \\
\hline & No psicotropa, $\mathrm{N}^{\circ}(\%)$ & $49(89,1)$ \\
\hline \multicolumn{2}{|c|}{ Dosis diaria media VR, mg (rango) } & $90(75-225)$ \\
\hline \multicolumn{2}{|c|}{ EVA- alcohol, puntuación media ${ }^{a}$} & $26,7 \pm 4,6$ \\
\hline \multicolumn{2}{|c|}{ EVA- cocaína, puntuación media ${ }^{a}$} & $6,9 \pm 3,0$ \\
\hline \multicolumn{2}{|c|}{ HAM-D ${ }_{17}$ puntuación media ${ }^{a}$} & $16,1 \pm 1,0$ \\
\hline \multirow{3}{*}{$\begin{array}{l}\text { ICG-S } \\
N^{\circ}(\%)\end{array}$} & Moderadamente enfermo & $7(12.7)$ \\
\hline & Marcadamente enfermo & $34(61,8)$ \\
\hline & Gravemente enfermo & $14(25,4)$ \\
\hline
\end{tabular}

a Media \pm desviación estándar; EVA: escala visual analógica; HAM-D ${ }_{17}$ : escala de Hamilton de Depresión de 17 ítems; ICG-S: Impresión Clínica Global severidad.

puntuación basal y final de 8,2 $\pm 0,2$ y 6,4 $\pm 0,4$, respectivamente $(P<0,01)$; área 5 , relaciones familiares/sociales, con una puntuación inicial de 6,9 $\pm 0,2$ y de $5,2 \pm 0,5$ en la visita mes $6^{\circ}(P<0,001)$; área 1 , situación médica, con puntuaciones de 3,7 $\pm 0,4$ y $0,9 \pm 0,3$ (visitas basal y mes $6^{\circ}$, respectivamente) $(P<0,001)$; y área 6 , situación psiquiátrica, con una puntuación basal de 7,8 $\pm 0,1$ y final de 5,4 \pm 0,4 ( $P$ $<0,001)$, donde se observó una mejoría significativa en los ítems analizados individualmente (ítems 3-9 del EuropASI). Se observaron también reducciones en las puntuaciones medias correspondientes al área 2 , empleo/soportes $\left(-0,8\right.$ puntos, mes $6^{\circ} \mathrm{vs}$ basal) y área 4, situación legal $\left(-0,7\right.$ puntos, mes $6^{\circ}$ vs basal), si bien no se alcanzaron diferencias significativas. En la figura 1 se refieren las puntuaciones obtenidas en el EuropASI en las distintas áreas analizadas, mientras que en la figura 2 se resumen las puntuaciones obtenidas en los 6 ítems del área psiquiátrica.

Las puntuaciones en la EVA de "craving" de alcohol descendieron significativamente a lo largo del estudio. Así, la puntuación en la visita basal fue de $26,7 \pm 4,6$, descendiendo a 4,1 $\pm 1,5$ en la visita final $(P<0,001)$ (figura 3$)$. En el caso del deseo de consumo de cocaína, se observó una reducción en la puntuación a lo largo del estudio, si bien no se observaron diferencias significativas entre la visita final y la basal (figura 3). El porcentaje de enfermos con con-

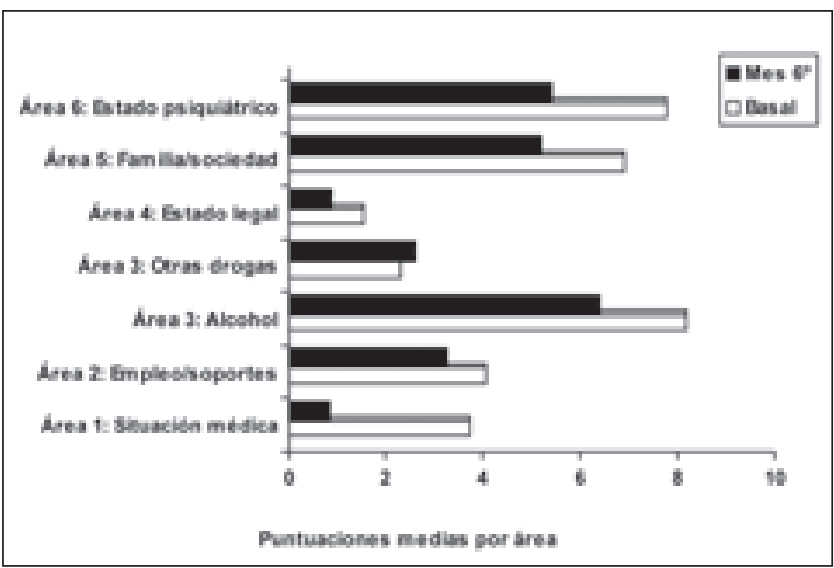

Figura 1.- Puntuaciones medias en el EuropASI. Visitas basal y mes $6^{\circ}$

EuropASI: Índice Europeo de Severidad Adicción. $P<0,01$, área 3; $P<0,001$, área 1,5 y 6 . Test de Wilcoxon.

sumo activo de alcohol disminuyó desde un 100\% en la visita basal hasta un $16,1 \%$ en la visita al mes $6^{\circ}$ $(P<0,001)$. En el caso del consumo de cocaína, bajó desde un $20 \%$ hasta un $6,5 \%$ en la visita al mes $6^{\circ}$ ( $P$ $<0,05)$. Al final del estudio, la detección de tóxicos en orina fue positiva en 3 pacientes $(9,7 \%$ sobre el total de la muestra); de estos, 2 casos fueron positivos para cocaína y un caso para cannabis. 


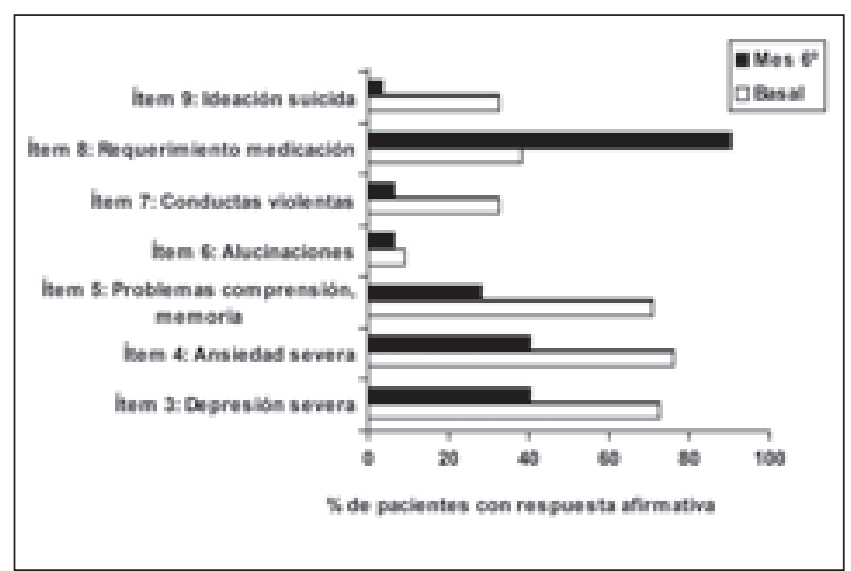

Figura 2.- Estado psiquiátrico según EuropASI. Visitas basal y mes $6^{\circ}$

EuropASI: Índice Europeo de Severidad Adicción. $P<0,05$, ítems 4 y 9; $P<0,01$, ítems 3, 5 y 7 ; y $P<0,001$, ítem 8; visita mes $6^{\circ}$ vs. basal. Test de $\mathrm{Q}$ de Cochran.

La puntuación media en la escala de HAM- $D_{17}$ en la visita basal fue de 16,1 $\pm 1,0$. Esta puntuación se redujo a lo largo del estudio hasta un 6,5 $\pm 1,1$ en la visita final $(P<0,001)$. También se obtuvieron diferencias significativas en el descenso de la puntuación al final del estudio en el caso del ítem 1 (humor deprimido) $(P=0,001)$, ítem 2 (ideaciones suicidas) $(P=$ 0,001 ), ítem 10 (ansiedad psíquica) $(P=0,001)$ e ítem 11 (ansiedad somática) $(P=0,002)$.

El estado del paciente, según la puntuación en la escala ICG-M, mejoró a lo largo del estudio. Los datos referidos por el investigador reflejan que el $81,2 \%$ de los pacientes puntuaron como "mucho/muchísimo mejor" al final del estudio $(P=0,002)$ (tabla 2). En el caso de la opinión del paciente, este porcentaje fue del $85,2 \%$. Al finalizar los 6 meses de tratamiento, se observó una reducción significativa en el consumo de fármacos psicotropos $(P=0,047)$, así como específi-

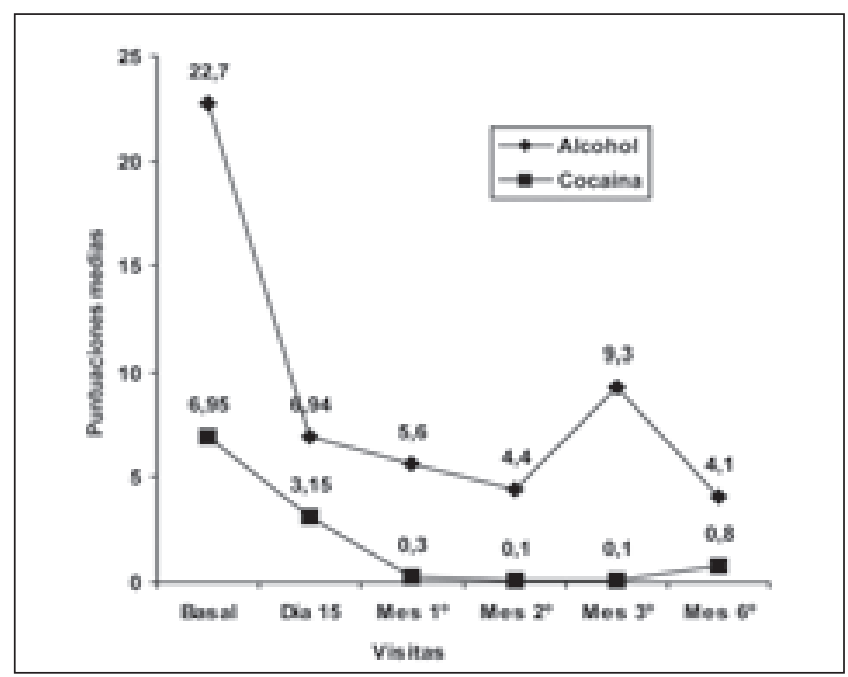

Figura 3.- Puntuaciones medias EVA para "craving" de alcohol y cocaína. Vistas basal y mes $6^{\circ}$.

EVA: Escala Visual Analógica. $P<0,001$, visita mes $6^{\circ}$ vs basal (EVA alcohol). Test de Wilcoxon.

camente en el consumo de ansiolíticos/hipnóticos ( $P$ $<0,001)$.

Al final del estudio se observó una reducción significativa en la medicación psicotropa concomitante administrada ( $P<0,01 \mathrm{vs}$. basal). Así, un $58,1 \%$ de los pacientes continuaban recibiendo medicación psicotropa, siendo los fármacos más frecuentemente administrados naltrexona (19,3\%), topiramato y lorazepan (9,7\%, ambos fármacos) (porcentajes sobre el total de la muestra). Un $61,3 \%$ de los pacientes continuaban recibiendo concomitantemente otra medicación no psicotropa, siendo los fármacos más frecuentemente administrados cianamida cálcica, disulfiram y ácido fólico $(54,8 \%, 25,8 \%$ y $9,7 \%$, respectivamente) La dosis media diaria de venlafaxina retard administrada en la visita final fue de $85,9 \mathrm{mg}$.

Un total de 21 pacientes $(38,2 \%)$ no terminaron el estudio; en total, 18 pacientes $(32,7 \%)$ discontinuaron por pérdidas en el seguimiento, 2 pacientes

Tabla 2: Evolución del estado general del paciente, conforme valoración del médico y paciente. Escala ICG-M.

\begin{tabular}{|c|c|c|c|c|}
\hline \multirow{2}{*}{ ICG-M } & \multicolumn{2}{|c|}{$\begin{array}{l}\text { Valoración del investigador* } \\
N^{\circ}(\%)\end{array}$} & \multicolumn{2}{|c|}{$\begin{array}{l}\text { Valoración del paciente } \\
N^{\circ}(\%)\end{array}$} \\
\hline & $\begin{array}{l}\text { Día } 15 \\
N=53\end{array}$ & $\begin{array}{l}\text { Mes } 6^{\circ} \\
N=27\end{array}$ & $\begin{array}{l}\text { Día } 15 \\
N=53\end{array}$ & $\begin{array}{l}\text { Mes } 6^{\circ} \\
N=27\end{array}$ \\
\hline Mínimamente peor & $1(1.9)$ & 0 & $1(1.9)$ & $01(2,4)$ \\
\hline Sin cambios & $2(3,8)$ & $2(7,4)$ & $2(3,8)$ & $1(3,7)$ \\
\hline Mínimamente mejor & $27(50,9)$ & $3(11,1)$ & $14(26,4)$ & $3(11,1)$ \\
\hline Mucho mejor & $22(41,5)$ & $20(74,2)$ & $27(50,9)$ & $18(66,7)$ \\
\hline Muchísimo mejor & $1(1,9)$ & $2(7,4)$ & $9(17)$ & $5(18,5)$ \\
\hline
\end{tabular}

ICG-M: Impresión Clínica Global mejoría. ${ }^{*} P=0,002$, visita mes $6^{\circ}$ vs. día 15 . Test de Friedman. 
$(3,6 \%)$ se retiraron por efectos adversos y 1 paciente $(1,8 \%)$ refirió otras causas. Un total de 7 pacientes $(12,7 \%)$ notificaron efectos adversos a lo largo del estudio. Los efectos adversos referidos fueron disfunción orgásmica (3 pacientes, 5,4\%), disminución del deseo sexual (2 pacientes; 3,6\%), disfunción eréctil (1 paciente, 1,8\%) y disfunción global de la función sexual (1 paciente, 1,8\%). La gravedad fue considera- da como leve-moderada en 4 casos $(7,3 \%)$ y severa en 3 casos (5,5\%). Todos los acontecimientos adversos se consideraron como posible o probablemente relacionados con el tratamiento del estudio. No se observaron modificaciones clínicamente significativas a lo largo del estudio en el peso ni en otros parámetros de seguridad cardiovasculares, tales como la tensión arterial y la frecuencia cardiaca (tabla 3).

Tabla 3.- Parámetros cardiovasculares: valores medios de TAS, TAD y FC.

\begin{tabular}{|c|c|c|c|}
\hline \multirow[b]{2}{*}{ VISITAS } & \multicolumn{3}{|c|}{ VARIABLES ANALIZADAS } \\
\hline & $\begin{array}{c}\text { TAS. } \mathrm{mm} \mathrm{Hg} \\
\text { Media } \pm \text { DE } \\
\text { (mediana; rango) }\end{array}$ & $\begin{array}{c}\text { TAD. } \mathrm{mm} \mathrm{Hg} \\
\text { Media } \pm \text { DE } \\
\text { (mediana; rango) }\end{array}$ & $\begin{array}{c}\text { FC (I.p.m) } \\
\text { Media } \pm \text { DE } \\
\text { (mediana; rango) }\end{array}$ \\
\hline Basal & $\begin{array}{c}118,3 \pm 1,7 \\
(120 ; 95-160)\end{array}$ & $\begin{array}{c}72,7 \pm 1,3 \\
(70 ; 60-100)\end{array}$ & $\begin{array}{c}81,3 \pm 1,5 \\
(80 ; 60-108)\end{array}$ \\
\hline Día 15 & $\begin{array}{c}115,9 \pm 1,8 \\
(115 ; 90-173)\end{array}$ & $\begin{array}{c}72 \pm 1,4 \\
(70 ; 50-92)\end{array}$ & $\begin{array}{c}81,4 \pm 1,4 \\
(80 ; 60-106)\end{array}$ \\
\hline Mes $1^{\circ}$ & $\begin{array}{c}118,6 \pm 2,0 \\
(120 ; 95-150)\end{array}$ & $\begin{array}{c}75,1 \pm 1,5 \\
(75 ; 60-100)\end{array}$ & $\begin{array}{c}79,6 \pm 1.4 \\
(80 ; 60-100)\end{array}$ \\
\hline $\operatorname{Mes} 2^{\circ}$ & $\begin{array}{c}118,3 \pm 2,4 \\
(120 ; 90-170)\end{array}$ & $\begin{array}{c}75,7 \pm 2,4 \\
(72,5 ; 60-160)\end{array}$ & $\begin{array}{c}77,5 \pm 1,5 \\
(80 ; 56-92)\end{array}$ \\
\hline Mes $3^{\circ}$ & $\begin{array}{c}117,5 \pm 2,8 \\
(120 ; 90-190)\end{array}$ & $\begin{array}{c}75,1 \pm 1,6 \\
(72,5 ; 50-100)\end{array}$ & $\begin{array}{c}79,1 \pm 1,6 \\
(80 ; 48-100)\end{array}$ \\
\hline Mes $6^{\circ}$ & $\begin{array}{c}112,4 \pm 2,2 \\
(110 ; 90-140)\end{array}$ & $\begin{array}{c}73,8 \pm 2 \\
(70 ; 60-95)\end{array}$ & $\begin{array}{c}78,3 \pm 2 \\
(80 ; 60-112)\end{array}$ \\
\hline
\end{tabular}

TAS: tensión arterial sistólica. TAD: tensión arterial diastólica. FC: frecuencia cardiaca. DE: desviación estándar.

\section{DISCUSIÓN}

Uno de los problemas sin resolver en el tratamiento del alcoholismo es el papel que deben jugar los antidepresivos ${ }^{26}$. Mientras que unos estudios han sugerido que estos tratamientos pueden ser efecti$\operatorname{vos}^{27,28}$, otros han obtenido resultados negativos ${ }^{29}$, 30. Los pacientes de este estudio acuden a una unidad de desintoxicación y permanecen ingresados aproximadamente doce-quince días para ser tratados de los síntomas agudos de la abstinencia y posteriormente son seguidos de forma ambulatoria. De manera concomitante al tratamiento con los fármacos más habitualmente utilizados para la desintoxicación y deshabituación, se añade venlafaxina retard. Estos fármacos son el clormetiazol, clorazepato y lorazepam además de disuasivos, vitaminas y fármacos neuroprotectores en este estudio. A la hora de interpretar los resultados debemos reseñar que los primeros quince días de tratamiento el seguimiento del paciente se realiza en el ámbito hospitalario, por lo que el contacto es continuo y su vigilancia muy estrecha.
Posteriormente las visitas fueron más espaciadas y de forma ambulatoria.

El tratamiento continuado con venlafaxina retard durante 24 semanas se asoció significativamente con una mejoría de la gravedad de la adicción, con una reducción de la necesidad de tratamiento para los problemas relacionados con el alcohol, así como con una mejoría global, percibida tanto por los clínicos como por los propios pacientes. Además los pacientes refirieron una disminución en el deseo de consumir alcohol (craving). Estos resultados, similares a los observados en pacientes alcohólicos deprimidos que recibieron venlafaxina ${ }^{14,15}$, indicarían que la venlafaxina podría tener un papel en el tratamiento de la dependencia de alcohol en pacientes que están siguiendo un tratamiento de deshabituación.

Los datos observados en pacientes consumidores de cocaína, no pasan de ser anecdóticos debido al número tan pequeño de pacientes. A pesar de que se observa un aumento en la puntuación del EuropASI en "otras drogas" se objetiva una disminución en el "craving" asociado al consumo, pero sin alcanzar 
significación estadística. En el estudio de McDowe$\|^{12}$, el autor discute que probablemente los pacientes cocainómanos informan a la baja su consumo, por lo que sería importante realizar estudios de laboratorio que nos confirmen el consumo activo. En nuestro estudio cabe destacar que tenemos los datos referidos de consumo de cocaína y de muestras de orina, no detectándose notificaciones a la baja; el número de pacientes que refieren consumo activo, concuerda con un resultado positivo en las muestras de orina.

Por otra parte, venlafaxina retard fue bien tolerada por los pacientes. Se debe destacar en este sentido que los pacientes reciben múltiples fármacos y no se observan problemas significativos de tolerancia o de interacciones con venlafaxina retard. Curiosamente el tipo de acontecimientos adversos no concuerda con los referidos en estudios previos en pacientes deprimidos $^{31}$. En general los más frecuentemente notificados en otros estudios son náuseas, epigastralgia, cefaleas, disminución de la libido, mareo y somnolencia. En nuestro estudio el 12,7\% de los pacientes comunicaron efectos adversos relacionados con la esfera sexual. Hay que destacar que la mayoría de los pacientes toman otras medicaciones psicotropas y no psicotropas a lo largo del estudio, con lo cual es difícil atribuirlos directamente a venlafaxina retard.

Una importante limitación de nuestro estudio es que al inicio del mismo no se descartó la existencia de un trastorno depresivo de manera concomitante. Solo disponemos de los datos cuantitativos de las puntuaciones en la escala HAM-D ${ }_{17}$, que disminuyeron desde $16,1 \pm 1,0$ en la visita basal hasta 6,5 $\pm 1,1$ en la visita final. Los pacientes pasaron según esta escala de presentar sintomatología leve a no presentar prácticamente síntomas. Al no haberse descartado la existencia de un episodio depresivo, no podemos ser categóricos al interpretar los resultados, ya que la mejoría en la sintomatología relacionada con el alcohol, podría ser debida al efecto antidepresivo ejercido por la venlafaxina retard sobre la sintomatología afectiva.

Otras importantes limitaciones de este estudio son el carácter observacional del mismo, el pequeño tamaño de la muestra, la elevada tasa de discontinuaciones y la falta de un control con placebo. No se puede descartar que la variable principal objeto del estudio pueda estar influenciada por diversos factores, tales como los efectos mediados por la medicación concomitante, la ayuda psicológica o la hospitalización, entre otros. Por todo ello, se debe tener precaución a la hora de interpretar los resultados.

En resumen, los resultados de este estudio abierto y observacional sugieren que la venlafaxina retard podría ser efectiva en el tratamiento coadyuvante de pacientes con dependencia de alcohol que estén realizando un tratamiento de deshabituación alcohólica, objetivándose como el fármaco podría contribuir a la mejora de los problemas relacionados con la dependencia de alcohol, detectados mediante el EuropASI. No obstante, estos resultados prometedores deberían ser replicados con estudios sobre series más amplias y controlados con placebo.

\section{REFERENCIAS}

1. Mason BJ, Kocsis JH, Ritvo EC, Cutler RB: A doubleblind, placebo-controlled trial of desipramine for primary alcohol dependence stratified on the presence or absence of major depression. JAMA 1996; 275: 761-7.

2. Holder HD, Wallack L. Contemporary perspectives for preventing alcohol problems: an empirically-derived model. J Public Health Policy 1986; 7: 324-39.

3. Fuller MG. A new day: strategies for managing psychiatric and substance abuse benefits. Health Care Manage Rev 1994; 19: 20-4.

4. Thase ME. Efficacy and tolerability of one daily venlafaxine extended release in outpatients with major deppression. J Clin Psychiatry 1997; 58: 393-398.

5. Lecrubier Y, Bourin M, Moon CA, Schifano F, Blanchard C, Danjou P, et al. Efficacy of venlafaxine in depressive illness in general practise. Acta Psychiatr Scand 1997; 95: 458-463.

6. Rickels K, Pollack MH, Sheehan DV, Haskins JT. Efficacy of extended-release venlafaxine in nondepressed outpatients with generalized anxiety disorder. Am J Psychiatry 2000; 157: 969-974.

7. Allgulander $C$, Hackett D, Salinas E. Venlafaxine extended release (ER) in the treatment of generalised anxiety disorder. Br J Psychiatr 2001; 179: 15-22.

8. Silverstone $\mathrm{PH}$, Salinas E. Efficacy of venlafaxine extended release in patients with major depressive disorder and comorbid generalized anxiety disorder. J Clin Psychiatry 2001; 62: 523-529.

9. Rudolph, RL. A meta anlysis of the effects of venlafaxine on anxiety associated with depression. J. Clin Psychol 1998; 18: 136-144.

10. Khan A, Upton GV, Rudolph RL, Entsuah R, Leventer $\mathrm{SM}$. The use of venlafaxine in the treatment of major depression and major depression associated with anxiety: a dose response study. J Clin Psychopharmacology 1998; 18: $18-25$.

11. Silverstone $\mathrm{PH}$ and Ravindran $\mathrm{A}$, for the venlafaxine XR 360 Study group. Once daily venlafaxine extended release (XR) compared with fluoxetine in outpatients with depression and anxiety. J Clinic Psychiatry 1999; 60: 1.

12. McDowell DM, Levin FR, Seracini AM, Nunes EV. Venlafaxine treatment of cocaine abusers with depressive disorders. Am J Drug Alcohol Abuse 2000; 26: 25-31. 
13. Upadhyaya HP, Brady KT, Sethuraman G, Sonne SC, Malcolm R. Venlafaxine treatment of patients with comorbid alcohol/cocaine abuse and attention-deficit/ hyperactivity disorder: a pilot study. J Clin Psychopharmacol 2001; 21: 116-8.

14. Bobes J, Saiz PA, Bousoño M. Venlafaxine in the treatment of depression in alcoholic patients. 21st Congress of the Collegium Internationale NeuroPsychophamacologium. 1997(Abstract PW 16008).

15. Garcia Portilla MP, Bascarán MT, Saiz PA, Mateos M, Gonzalez-Quirós M, Perez P, et al. Efectividad de la venlafaxina en el tratamiento de la dependencia de alcohol con depresión comórbida. Actas Esp Psiquiatr 2005; 33: 41-5.

16. Foltin RW, Ward AS, Collins ED, Haney M, Hart CL, Fischman MW. The effects of venlafaxine on the subjective, reinforcing, and cardiovascular effects of cocaine in opioid-dependent and non-opioid-dependent humans. Exp Clin Psychopharmacol 2003; 11: 123-30.

17. American Psychiatric Association. Diagnostic and Statistical Manual of Mental Disorders, Fourth Edition. Washington DC: American Psychiatric Association. 1994.

18. Kokkevi A, Hartgers C. European adaptacion of a multidimensional assessment instrument for drug and alcohol dependence. Eur Addict Res 1995; 1: 208-210.

19. Garcia-Portilla, MP, Saiz Martinez PA, Bousoño Garcia M, Bobes Garcia J. Evaluación de la gravedad de la conducta alcohólica. Psiquiatría Biológica. 1998; 5: 40-43.

20. DeLoach LJ, Higgins MS, Caplan AB, Stiff JL. The visual analog scale in the immediate postoperative period: intrasubject variability and correlation with a numeric scale. Anesth Analg 1998; 86: 102-6.
21. Bodian CA, Freedman G, Hossian S, Eisenkraft JB, Beilin $Y$. The visual analog scale for pain: clinical significance in postoperative patients. Anesthesiology 2001; 95: 1356-61.

22. Bech P. Rating scales for psychopathology, Health status, and quality of life. Berlin: Springer-Verlag, 1993: 333: 35.

23. Hamilton $M$. Development of a rating scale for primary depressive illness. Br J Soc Clin Psychol 1967; 6: 278-296.

24. Ramos Brieva JA, Cordero Villafáfila A. A new validation of the Hamilton Rating scale for depression. J Psychistric Research 1988; 22: 21-28.

25. Hedlun JL, Vieweg BW. The Hamilton rating scale for depression: A comprehensive review. J Operat Psychiatry 1979; 10: 149-165.

26. Petrakis IL, Leslie D, Rosenheck R. The use of antidepressants in alcohol-dependent veterans. J Clin Psychiatry 2003; 64: 865-870.

27. Litte RZ, Allen JP. Phatmacotherapies for alcoholism: promising agents and clinical sigues. Alcohol Clin Exp Res 1991; 15: 620-633.

28. Pettinati HM, Volpicelli JR, Luck G, Kranzler HR, Rukstalis MR, Cnaan A. Double-blind clinical trial of sertraline treatment for alcohol dependence. J Clin Psychopharmacol 2001; 21: 143-153.

29. Kranzler HR, Burleson JA, Korner P, Del Boca FK, Bohn MJ, Brrown J. Placebo-controlled trial of fluoxetine as an adjunct to relapse prevention in alcoholics. Am J Psychiatry 1995; 152: 391-397.

30. Ciraulo DA, Jaffe JH. Tryciclic antidepressants in the treatment of depression associated with alcoholism. J Clin Psychopharmacol 1981; 1: 146-150.

31. Blythe D, Hackett LP. Cardiovascular and neurological toxicity of venlafaxine. Hum Exp Toxicol 1999; 18: 309-13 